\section{Getting around peripartum cardiomyopathy}

\section{By Michael J. Haas, Senior Writer}

A Harvard Medical School-led team has shown that a soluble form of VEGF receptor 1 causes about one-third of peripartum cardiomyopathy cases-those associated with pre-eclampsia. ${ }^{1}$ Ongoing studies are seeking to identify small molecule inhibitors of the protein and determine whether the mechanism also underlies the other two-thirds of cases.

Peripartum cardiomyopathy (PPCM) is a form of heart failure that affects about 1 in 1,000 peripartum women. The disease is usually diagnosed a few weeks postpartum, and current treatments are limited to standard therapies for heart failure such as $\beta$-blockers and angiotensin-converting enzyme (ACE) inhibitors.

A known risk factor for PPCM is preeclampsia, a condition involving hypertension and proteinuria that occurs in about $5 \%$ of all pregnant women but in about $33 \%$ of pregnant women with PPCM. The connection between the two diseases and the mechanism of PPCM is poorly understood.

The Harvard Medical School (HMS) team did not set out to investigate the cause of PPCM but instead stumbled upon it serendipitously while investigating the proangiogenic functions of peroxisome proliferationactivated receptor- $\gamma$ coactivator $1 \alpha$ (PPARGC1A; PGC- $1 \alpha$ ) in the heart.

The researchers were breeding heart-specific $P g c-1 \alpha$ knockout mice and noticed knockout females died after delivering only one or two litters, whereas wild-type females delivered five or more litters without incident. Compared with knockout males, or knockout females that had not yet been pregnant, the postpartum knockout females had enlarged, fibrotic hearts, decreased vascular density in the heart and other signs of cardiomyopathy.

Additional experiments in the knockout mice confirmed that lack of Pgc-1 $\alpha$ downregulated two proangiogenic pathways in the heart to cause PPCM. These findings led the group to speculate that human PPCM might also result from an angiogenic deficiency.

The researchers suspected the culprit was a soluble form of VEGF receptor 1 (FLT1; VEGFR-1). Soluble FLT1 (sFLT1) is an antiangiogenic factor normally secreted by the placenta in late pregnancy, resulting in increased serum levels during the peripartum period. However, the levels are much greater in peripartum women with pre-eclampsia than in healthy peripartum women. ${ }^{2}$

In healthy and most pre-eclampsic peripartum women, serum levels of sFLT1 return to normal, prepregnancy levels within three days after delivery.

The team found that at 4-6 weeks after delivery, sFLT1 levels were 5- to 10-fold higher in women who had pre-eclampsia and PPCM than in postpartum women who did not have either condition.

Next, the team showed that in prepartum women with pre-eclampsia, higher sFLT1 correlated with lower cardiac function as measured by echocardiography. Similar cardiac dysfunction, cardiomyopathy and heart failure occurred when murine sFlt1 was given to pregnant wildtype mice, nonpregnant wild-type mice and nonpregnant cardiacspecific $P g c-1 \alpha$ knockout mice.

Data were reported in Nature. ${ }^{1}$

Collectively, the findings suggest increased levels of sFLT1 cause PPCM that is associated with pre-eclampsia. The results also suggest that lowering sFLT1 levels could help treat PPCM, team leader Zoltan Arany told SciBX.

"We also think-but don't know yet-that the mechanism we describe in this study will apply to all cases of PPCM, not just the onethird associated with pre-eclampsia," he said.

Arany is assistant professor of medicine at HMS and a principal investigator at Beth Israel Deaconess Medical Center. The team included researchers from the Howard Hughes Medical Institute (HHMI) and Hannover Medical School.

\section{Flt to be tried}

Going forward, the challenges are identifying feasible sFLT1-reducing strategies and determining whether increased sFLT1 plays a causal role in the broader population of patients with PPCM.

Arany noted that last year, a group from HMS and Massachusetts General Hospital reported results from a pilot study showing that a dialysis-like device that removed circulating sFLT1 was successful in treating pre-eclampsia. ${ }^{3}$

That group was led by S. Ananth Karumanchi, coauthor on the current Nature study. Karumanchi is an associate professor of medicine at HMS, senior scientist at Beth Israel and investigator at HHMI.

"In theory, his device could also be used to treat PPCM," Arany said. "But another, less cumbersome way to treat the disease would be to use a systemic small molecule to inhibit sFLT1 secretion.”

Arany's team is now looking for such molecules. He said FLT1 inhibitors on the market to treat renal cancer and/or sarcoma-such as Votrient pazopanib from GlaxoSmithKline plc and Inlyta axitinib from Pfizer Inc. - are unlikely to be suitable for the PPCM indication. Those molecules, he noted, are intended to block activity of membrane-bound FLT1 as opposed to neutralizing the circulating, soluble form of the receptor.

sFLT1 is thought to play an important prepartum role by 


\section{ANALYSIS}

disconnecting the link between the mother's and baby's vascular systems - thereby protecting the mother from potentially fatal blood loss during childbirth. However, Arany does not think lowering sFLT1 to treat PPCM would raise major safety concerns because the indication is diagnosed after delivery.

He added that the source of increased postpartum sFLT1 is unknown but is thought to come from placental remnants, syncytial microparticles shed by the placenta or circulating mononuclear cells able to express the protein.

In addition to identifying inhibitors of sFLT1 secretion, Arany said the group is conducting serial blood tests and other prospective studies in pregnant women to investigate whether sFLT1 could be a marker or a therapeutic target in cases of PPCM not associated with pre-eclampsia.

Earlier this year, a team led by Karumanchi reported that sFLT1 could be a diagnostic and prognostic marker for pre-eclampsia. ${ }^{4}$ Current markers for the condition are high blood pressure and protein in the urine, but they do not predict outcomes.

If prepartum sFLT1 also proves to be a risk marker for PPCM, sFLT1reducing therapy might be used to prevent the disease, Arany said. "I think prevention in high-risk, prepartum women is a good approach and might even be better than treatment," he said.

Arany added that the caveat is that a prophylactic would have to reduce sFLT1 levels only to what is normal in prepartum women.

The findings reported in Nature are not patented and are available for partnering, Arany said.

Haas, M.J. SciBX 5(21); doi:10.1038/scibx.2012.538

Published online May 24, 2012

\section{REFERENCES}

1. Patten, I.S. et al. Nature; published online May 9, 2012; doi:10.1038/nature11040

Contact: Zoltan Arany, Harvard Medical School, Boston, Mass. e-mail: zarany@bidmc.harvard.edu

Contact: Denise Hilfiker-Kleiner, Hannover Medical School, Hannover, Germany e-mail: hilfiker.denise@mh-hannover.de

2. Levine, R.J. et al. N. Engl. J. Med. 350, 672-683 (2004)

3. Thadhani, R. et al. Circulation 124, 940-950 (2011)

4. Rana, S. et al. Circulation 125, 911-919 (2012)

\section{COMPANIES AND INSTITUTIONS MENTIONED}

Beth Israel Deaconess Medical Center, Boston, Mass. GlaxoSmithKline plc (LSE:GSK; NYSE:GSK), London, U.K. Hannover Medical School, Hannover, Germany Harvard Medical School, Boston, Mass. Howard Hughes Medical Institute, Chevy Chase, Md. Massachusetts General Hospital, Boston, Mass. Pfizer Inc. (NYSE:PFE), New York, N.Y. 